\title{
Some identities for umbral calculus associated with partially degenerate Bell numbers and polynomials
}

\author{
Taekyun Kim ${ }^{\mathrm{a}, \mathrm{b}}$, Dae San Kim ${ }^{\mathrm{c}}$, Hyuck-In Kwon ${ }^{\mathrm{b}}$, Seog-Hoon Rim ${ }^{\mathrm{d}, *}$ \\ ${ }^{a}$ Department of Mathematics, College of Science Tianjin Polytechnic University, Tianjin 300160, China. \\ ${ }^{b}$ Department of Mathematics, Kwangwoon University, Seoul 139-701, Republic of Korea. \\ ${ }^{c}$ Department of Mathematics, Sogang University, Seoul 121-742, Republic of Korea. \\ ${ }^{d}$ Department of Mathematics Education, Kyungpook National University, Taegu 702-701, Republic of Korea.
}

Communicated by Y. J. Cho

\begin{abstract}
In this paper, we study partially degenerate Bell numbers and polynomials by using umbral calculus. We give some new identities for these numbers and polynomials which are associated with special numbers and polynomial. (C)2017 All rights reserved.
\end{abstract}

Keywords: Partially degenerate Bell polynomials, umbral calculus.

2010 MSC: 05A19, 05A40, 11B83.

\section{Introduction}

The Bell polynomials (also called the exponential polynomials and denoted by $\phi_{n}(x)$ ) are defined by the generating function (see $[4,10,16]$ )

$$
e^{x\left(e^{t}-1\right)}=\sum_{n=0}^{\infty} \operatorname{Bel}_{n}(x) \frac{t^{n}}{n !} .
$$

From (1.1), we note that

$$
\begin{aligned}
& \operatorname{Bel}_{0}(x)=1, \operatorname{Bel}_{1}(x)=x, \operatorname{Bel}_{2}(x)=x^{2}+x, \operatorname{Bel}_{3}(x)=x^{3}+3 x^{2}+x \\
& \operatorname{Bel}_{4}(x)=x^{4}+6 x^{3}+7 x^{2}+x, \operatorname{Bel}_{5}(x)=x^{5}+10 x^{4}+25 x^{3}+15 x^{2}+x, \cdots .
\end{aligned}
$$

When $x=1, \operatorname{Bel}_{n}=\operatorname{Bel}_{n}(1)$ are called the Bell numbers. As is well-known, the Stirling numbers of the second kind are defined by the generating function (see $[12,14,16])$

$$
\left(e^{t}-1\right)^{m}=m ! \sum_{n=m}^{\infty} S_{2}(n, m) \frac{t^{n}}{n !},(m \geqslant 0) .
$$

\footnotetext{
*Corresponding author

Email addresses: tkkim@kw.ac.kr (Taekyun Kim), dskim@sogang.ac.kr (Dae San Kim), sura@kw.ac.kr (Hyuck-In Kwon), shrim@knu.ac.kr (Seog-Hoon Rim)

doi:10.22436/jnsa.010.06.11
}

Received 2017-03-25 
The Stirling numbers of the first kind are defined as (see [1-17])

$$
(x)_{n}=\sum_{l=0}^{n} S_{1}(n, l) x^{l}(n \geqslant 0),
$$

where $(x)_{0}=1,(x)_{n}=x(x-1) \cdots(x-n+1),(n \geqslant 1)$. From $(1.1)$, we note that $($ see $[10,12,16])$

$$
\operatorname{Bel}_{n}(x)=\sum_{l=0}^{n} S_{2}(n, l) x^{l},(n \geqslant 0) .
$$

The Bernoulli polynomials are given by the generating function as follows (see [1-17])

$$
\frac{t}{e^{t}-1} e^{x t}=\sum_{n=0}^{\infty} B_{n}(x) \frac{t^{n}}{n !} .
$$

When $x=0, B_{n}=B_{n}(x)$ are called Bernoulli numbers. From (1.2), we note that (see $\left.[7,11,12,16]\right)$

$$
B_{n}(x)=\sum_{l=0}^{n}\left(\begin{array}{l}
n \\
l
\end{array}\right) B_{l} x^{n-l} .
$$

Note that

$$
\frac{d}{d x} B_{n}(x)=n B_{n-1}(x),(n \geqslant 1) .
$$

Recently, Kim-Kim considered the partially degenerate Bell polynomials which are given by the generating function (see [13])

$$
e^{x\left((1+\lambda t)^{\frac{1}{\lambda}}-1\right)}=\sum_{n=0}^{\infty} \operatorname{Bel}_{n, \lambda}(x) \frac{t^{n}}{n !} .
$$

When $x=1, \operatorname{Bel}_{\mathfrak{n}, \lambda}=\operatorname{Bel}_{\mathfrak{n}, \lambda}(1)$ are called the partially degenerate Bell numbers. Note that $\lim _{\lambda \rightarrow 0} \operatorname{Bel}_{\mathfrak{n}, \lambda}(x)$ $=\operatorname{Bel}_{n}(x),(n \geqslant 0)$. Let $\mathbb{C}$ be the complex number field and let $\mathcal{F}$ be the set of all formal power series in variable $t$ over $\mathbb{C}$ with

$$
\mathcal{F}=\left\{f(t)=\sum_{k=0}^{\infty} a_{k} \frac{t^{k}}{k !} \mid a_{k} \in \mathbb{C}\right\} .
$$

Let $\mathbb{P}=\mathbb{C}[x]$ and let $\mathbb{P}^{*}$ be the vector space of all linear functionals on $\mathbb{P}$. The action of a linear functional $L \in \mathbb{P}^{*}$ on a polynomial $p(x)$ is denoted by $\langle L \mid p(x)\rangle$, which is linearly extended by the rule $\left\langle c L+c^{\prime} L^{\prime} \mid p(x)\right\rangle=c\langle L \mid p(x)\rangle+c^{\prime}\left\langle L^{\prime} \mid p(x)\right\rangle$, where $c, c^{\prime} \in \mathbb{C}$. For $f(t)=\sum_{k=0}^{\infty} a_{k} \frac{t^{k}}{k !} \in \mathcal{F}$, the action $\langle f(t) \mid \cdot\rangle$ of the linear functional $f(t)$ on $\mathbb{P}$ is defined by (see $[11,16]$ )

$$
\left\langle f(t) \mid x^{n}\right\rangle=a_{n} \text { for all } n \geqslant 0 .
$$

By (1.4), we easily get (see $[11,14,16])$

$$
\left\langle t^{k} \mid x^{n}\right\rangle=n ! \delta_{n, k},(n, k \geqslant 0),
$$

where $\delta_{n, k}$ is the Kronecker symbol. Let $f_{L}(t)=\sum_{k=0}^{\infty}\left\langle L \mid x^{k}\right\rangle \frac{t^{k}}{k !}$. Then, from (1.5), we have

$$
\left\langle f_{L}(t) \mid x^{n}\right\rangle=\left\langle L \mid x^{n}\right\rangle .
$$


Additionally, the map $L \longmapsto f_{L}(t)$ is a vector space isomorphism from $\mathbb{P}^{*}$ onto $\mathcal{F}$. Henceforth, $\mathcal{F}$ denotes both the algebra of the formal power series in $t$ and the vector space of all linear functionals on $\mathbb{P}$, and an element $f(t)$ of $\mathcal{F}$ will be thought of as both a formal power series and a linear functional. We call $\mathcal{F}$ umbral algebra. The umbral calculus is the study of umbral algebra (see $[11,14,16])$.

Let $f(t)(\neq 0) \in \mathcal{F}$. Then the order of $f(t)$ is the smallest positive integer $k$ for which the coefficient of $t^{k}$ does not vanish. The order of $f(t)$ is denoted by $o(f(t))$. Let $f(t), g(t) \in \mathcal{F}$ with $o(f(t))=1$ and $o(g(t))=0$. Then there exists a unique sequence $S_{n}(x)$ of polynomials such that

$$
\left\langle g(t) f(t)^{k} \mid S_{n}(x)\right\rangle=\left\langle g(t) \mid f(t)^{k} S_{n}(x)\right\rangle=n ! \delta_{n, k},(n, k \geqslant 0), \quad(\text { see [16]). }
$$

The sequence $S_{n}(x)$ is called the Sheffer sequence for $(g(t), f(t))$, for which we write $S_{n}(x) \sim(g(t), f(t))$.

Let $f(t)=\sum_{k=0}^{\infty} a_{k} \frac{t^{k}}{k !} \in \mathcal{F}$ and $p(x) \in \mathbb{P}$. Then, by (1.5), we get

$$
f(t)=\sum_{k=0}^{\infty}\left\langle f(t) \mid x^{k}\right\rangle \frac{t^{k}}{k !}, \quad p(x)=\sum_{k=0}^{\infty}\left\langle t^{k} \mid p(x)\right\rangle \frac{x^{k}}{k !} .
$$

From (1.6), we have

$$
p^{(k)}(0)=\left\langle t^{k} \mid p(x)\right\rangle=\left\langle 1 \mid p^{(k)}(x)\right\rangle, p^{(k)}(x)=\left(\frac{d}{d x}\right)^{k} p(x)
$$

By (1.7), we easily get

$$
t^{k} p(x)=p^{(k)}(x), e^{y t} p(x)=p(x+y), \text { and }\left\langle e^{y t} \mid p(x)\right\rangle=p(y) .
$$

It is well-known that

$$
S_{n}(x) \sim(g(t), f(t)) \Longleftrightarrow \frac{1}{g(\bar{f}(t))} e^{x \bar{f}(t)}=\sum_{n=0}^{\infty} S_{n}(x) \frac{t^{n}}{n !}, \quad(\text { see }[16]),
$$

where $\bar{f}(t)$ is the compositional inverse of $f(t)$ satisfying $\bar{f}(f(t))=f(\bar{f}(t))=t$. Let $f_{1}(t), f_{2}(t), \cdots, f_{m}(t) \in$ $\mathcal{F}$. Then we have

$$
\left\langle f_{1}(t) \cdots f_{m}(t) \mid x^{n}\right\rangle=\sum_{i_{1}+\cdots+i_{m}=n}\left(\begin{array}{c}
n \\
i_{1}, \cdots, i_{m}
\end{array}\right)\left\langle f_{1}(t) \mid x^{i_{1}}\right\rangle \cdots\left\langle f_{m}(t) \mid x^{i_{m}}\right\rangle .
$$

Let $f(t)$ be the linear functional such that

$$
\langle f(t) \mid p(x)\rangle=\int_{0}^{y} p(u) d u
$$

for all polynomials $p(x)$. Then, from (1.6), we have

$$
f(t)=\sum_{k=0}^{\infty} \frac{\left\langle f(t) \mid x^{k}\right\rangle}{k !} t^{k}=\sum_{k=0}^{\infty} \frac{y^{k+1}}{(k+1) !} t^{k}=\frac{1}{t}\left(e^{y t}-1\right) .
$$

Thus, by (1.10) and (1.11), we get

$$
\left\langle\frac{e^{y t}-1}{t} \mid p(x)\right\rangle=\int_{0}^{y} p(u) d u, \quad \frac{e^{y t}-1}{t} p(x)=\int_{x}^{x+y} p(u) d u .
$$

For $S_{n}(x) \sim(g(t), f(t))$ and $r_{n}(x) \sim(h(t), l(t))$, we have (see $\left.[11,14,16]\right)$

$$
S_{n}(x)=\sum_{k=0}^{n} C_{n, k} r_{k}(x)
$$


where

$$
C_{n, k}=\frac{1}{k !}\left\langle\frac{h(\bar{f}(t))}{g(\bar{f}(t))}(l(\bar{f}(t)))^{k} \mid x^{n}\right\rangle .
$$

In this paper, we study partially degenerate Bell numbers and polynomials by using umbral calculus. We give some new identities for these numbers and polynomials which are associated with special numbers and polynomials.

\section{Some identities for the partially degenerate Bell numbers and polynomials}

From (1.3), we recall that

$$
e^{x\left((1+\lambda t)^{\frac{1}{\lambda}}-1\right)}=\sum_{n=0}^{\infty} \operatorname{Bel}_{n, \lambda}(x) \frac{t^{n}}{n !}
$$

Note that

$$
\sum_{n=0}^{\infty} \lim _{\lambda \rightarrow 0} \operatorname{Bel}_{n, \lambda}(x) \frac{t^{n}}{n !}=\lim _{\lambda \rightarrow 0} e^{x\left((1+\lambda t)^{\frac{1}{\lambda}}-1\right)}=e^{x\left(e^{t}-1\right)}=\sum_{n=0}^{\infty} \operatorname{Bel}_{n}(x) \frac{t^{n}}{n !} .
$$

By (2.1), we get

$$
\lim _{\lambda \rightarrow 0} \operatorname{Bel}_{n, \lambda}(x)=\operatorname{Bel}_{n}(x),(n \geqslant 0) .
$$

From (1.9), we note that

$$
\operatorname{Bel}_{n, \lambda}(x) \sim\left(1, \frac{(1+t)^{\lambda}-1}{\lambda}\right) \Longleftrightarrow e^{x\left((1+\lambda t)^{\frac{1}{\lambda}}-1\right)}=\sum_{n=0}^{\infty} \operatorname{Bel}_{n, \lambda}(x) \frac{t^{n}}{n !} .
$$

Now, we observe that

$$
\begin{aligned}
\sum_{n=0}^{\infty} \operatorname{Bel}_{n, \lambda}(x) \frac{t^{n}}{n !} & =e^{x\left((1+\lambda t)^{\frac{1}{\lambda}}-1\right)}=\sum_{m=0}^{\infty} \frac{x^{m}}{m !}\left((1+\lambda t)^{\frac{1}{\lambda}}-1\right)^{m} \\
& =\sum_{m=0}^{\infty} \frac{x^{m}}{m !}\left(e^{\frac{1}{\lambda} \log (1+\lambda t)}-1\right)^{m} \\
& =\sum_{m=0}^{\infty} x^{m} \sum_{k=m}^{\infty} S_{2}(k, m) \lambda^{-k} \frac{1}{k !}(\log (1+\lambda t))^{k} \\
& =\sum_{k=0}^{\infty}\left(\sum_{m=0}^{k} S_{2}(k, m) x^{m} \lambda^{-k}\right) \frac{1}{k !}(\log (1+\lambda t))^{k} \\
& =\sum_{k=0}^{\infty}\left(\sum_{m=0}^{k} S_{2}(k, m) x^{m} \lambda^{-k}\right) \sum_{n=k}^{\infty} S_{1}(n, k) \lambda^{n} \frac{t^{n}}{n !} \\
& =\sum_{n=0}^{\infty}\left(\sum_{k=0}^{n} \sum_{m=0}^{k} S_{2}(k, m) S_{1}(n, k) x^{m} \lambda^{n-k}\right) \frac{t^{n}}{n !}
\end{aligned}
$$

Comparing the coefficients on both sides of (2.3), we obtain the following theorem.

Theorem 2.1. For $\mathrm{n} \geqslant 0$, we have

$$
\operatorname{Bel}_{n, \lambda}(x)=\sum_{k=0}^{n} \sum_{m=0}^{k} S_{2}(k, m) S_{1}(n, k) \lambda^{n-k} x^{m}=\sum_{m=0}^{n}\left(\sum_{k=m}^{n} S_{2}(k, m) S_{1}(n, k) \lambda^{n-k}\right) x^{m} .
$$


By (1.12), we get

$$
\begin{aligned}
\frac{e^{t}-1}{t} \operatorname{Bel}_{n, \lambda}(x) & =\int_{x}^{x+1} \operatorname{Bel}_{n, \lambda}(u) d u \\
& =\sum_{k=0}^{n} \sum_{m=0}^{k} S_{2}(k, m) S_{1}(n, k) \lambda^{n-k} \int_{x}^{x+1} u^{m} d u \\
& =\sum_{k=0}^{n} \sum_{m=0}^{k} S_{2}(k, m) S_{1}(n, k) \lambda^{n-k} \frac{1}{m+1}\left((x+1)^{m+1}-x^{m+1}\right) \\
& =\sum_{k=0}^{n} \sum_{m=0}^{k} \sum_{l=0}^{m}\left(\begin{array}{c}
m+1 \\
l
\end{array}\right) \frac{1}{m+1} S_{2}(k, m) S_{1}(n, k) \lambda^{n-k} x^{l} \\
& =\sum_{l=0}^{n}\left(\sum_{k=l}^{n} \sum_{m=l}^{k}\left(\begin{array}{c}
m+1 \\
l
\end{array}\right) \frac{1}{m+1} S_{2}(k, m) S_{1}(n, k) \lambda^{n-k}\right) x^{l}
\end{aligned}
$$

From (1.2) and (1.9), we have

$$
B_{n}(x) \sim\left(\frac{e^{t}-1}{t}, t\right) \Longleftrightarrow \frac{t}{e^{t}-1} e^{x t}=\sum_{n=0}^{\infty} B_{n}(x) \frac{t^{n}}{n !} .
$$

Thus, by (2.5), we get

$$
B_{n}(x)=\frac{t}{e^{t}-1} x^{n},(n \geqslant 0) .
$$

From (2.4) and (2.6), we can derive the following equation (2.7):

$$
\begin{aligned}
\operatorname{Bel}_{n, \lambda}(x) & =\sum_{k=0}^{n} \sum_{m=0}^{k} \sum_{l=0}^{m}\left(\begin{array}{c}
m+1 \\
l
\end{array}\right) S_{2}(k, m) S_{1}(n, k) \lambda^{n-k} \frac{1}{m+1} \frac{t}{e^{t}-1} x^{l} \\
& =\sum_{k=0}^{n} \sum_{m=0}^{k} \sum_{l=0}^{m}\left(\begin{array}{c}
m+1 \\
l
\end{array}\right) S_{2}(k, m) S_{1}(n, k) \lambda^{n-k} \frac{1}{m+1} B_{l}(x) .
\end{aligned}
$$

Therefore, by (2.7), we obtain the following theorem.

Theorem 2.2. For $\mathrm{n} \geqslant 0$, we have

$$
\operatorname{Bel}_{n, \lambda}(x)=\sum_{k=0}^{n} \sum_{m=0}^{k} \sum_{l=0}^{m}\left(\begin{array}{c}
m+1 \\
l
\end{array}\right) S_{2}(k, m) S_{1}(n, k) \lambda^{n-k} \frac{1}{m+1} B_{l}(x) .
$$

By (1.8) and (2.7), we get

$$
\begin{aligned}
\operatorname{tBel}_{n, \lambda}(x) & =\sum_{k=0}^{n} \sum_{m=0}^{k} \sum_{l=0}^{m}\left(\begin{array}{c}
m+1 \\
l
\end{array}\right) S_{2}(k, m) S_{1}(n, k) \lambda^{n-k} \frac{1}{m+1} \mathrm{tB}_{l}(x) \\
& =\sum_{k=1}^{n} \sum_{m=1}^{k} \sum_{l=1}^{m}\left(\begin{array}{c}
m+1 \\
l
\end{array}\right) S_{2}(k, m) S_{1}(n, k) \lambda^{n-k} \frac{l}{m+1} B_{l-1}(x) \\
& =\sum_{k=1}^{n} \sum_{m=1}^{k} \sum_{l=0}^{m-1}\left(\begin{array}{c}
m+1 \\
l+1
\end{array}\right) S_{2}(k, m) S_{1}(n, k) \lambda^{n-k} \frac{l+1}{m+1} B_{l}(x) \\
& =\sum_{k=1}^{n} \sum_{m=1}^{k} \sum_{l=0}^{m-1}\left(\begin{array}{c}
m \\
l
\end{array}\right) S_{2}(k, m) S_{1}(n, k) \lambda^{n-k} B_{l}(x) .
\end{aligned}
$$


From (1.12), we have

$$
\begin{aligned}
\frac{e^{y t}-1}{t} \operatorname{Bel}_{n, \lambda}(x)= & \int_{x}^{x+y} \operatorname{Bel}_{n, \lambda}(u) d u \\
= & \sum_{k=0}^{n} \sum_{m=0}^{k} \sum_{l=0}^{m}\left(\begin{array}{c}
m+1 \\
l
\end{array}\right) S_{2}(k, m) S_{1}(n, k) \lambda^{n-k} \frac{1}{m+1} \int_{x}^{x+y} B_{l}(u) d u \\
= & \sum_{k=0}^{n} \sum_{m=0}^{k} \sum_{l=0}^{m}\left(\begin{array}{c}
m+1 \\
l
\end{array}\right) S_{2}(k, m) S_{1}(n, k) \lambda^{n-k} \frac{1}{(m+1)(l+1)} \\
& \times\left(B_{l+1}(x+y)-B_{l+1}(x)\right) \\
= & \sum_{k=0}^{n} \sum_{m=0}^{k} \sum_{l=0}^{m} \sum_{j=0}^{l}\left(\begin{array}{c}
m+1 \\
l
\end{array}\right)\left(\begin{array}{c}
l+1 \\
j
\end{array}\right) S_{2}(k, m) S_{1}(n, k) \frac{\lambda^{n-k}}{(m+1)(l+1)} y^{l+1-j} B_{j}(x) .
\end{aligned}
$$

Thus, by (2.8), we get

$$
\begin{aligned}
\operatorname{Bel}_{n, \lambda}(x+y) & -\operatorname{Bel}_{n, \lambda}(x) \\
& =\left(e^{y t}-1\right) \operatorname{Bel}_{n, \lambda}(x) \\
& =\sum_{k=0}^{n} \sum_{m=0}^{k} \sum_{l=0}^{m} \sum_{j=0}^{l}\left(\begin{array}{c}
m+1 \\
l
\end{array}\right)\left(\begin{array}{c}
l+1 \\
j
\end{array}\right) S_{2}(k, m) S_{1}(n, k) \frac{\lambda^{n-k}}{(m+1)(l+1)} y^{l+1-j} t B_{j}(x) \\
& =\sum_{k=1}^{n} \sum_{m=1}^{k} \sum_{l=1}^{m} \sum_{j=1}^{l}\left(\begin{array}{c}
m+1 \\
l
\end{array}\right)\left(\begin{array}{c}
l+1 \\
j
\end{array}\right) S_{2}(k, m) S_{1}(n, k) \frac{j \lambda^{n-k}}{(m+1)(l+1)} y^{l+1-j} B_{j-1}(x) \\
& =\sum_{k=1}^{n} \sum_{m=1}^{k} \sum_{l=1}^{m} \sum_{j=0}^{l-1}\left(\begin{array}{c}
m+1 \\
l
\end{array}\right)\left(\begin{array}{c}
l+1 \\
j+1
\end{array}\right) S_{2}(k, m) S_{1}(n, k) \frac{(j+1) \lambda^{n-k}}{(m+1)(l+1)} y^{l-j} B_{j}(x) \\
& =\sum_{k=1}^{n} \sum_{m=1}^{k} \sum_{l=1}^{m} \sum_{j=0}^{l-1}\left(\begin{array}{c}
m+1 \\
l
\end{array}\right)\left(\begin{array}{c}
l \\
j
\end{array}\right) S_{2}(k, m) S_{1}(n, k) \frac{\lambda^{n-k}}{m+1} y^{l-j} B_{j}(x) .
\end{aligned}
$$

Therefore, by (2.9), we obtain the following theorem.

Theorem 2.3. For $\mathrm{n} \geqslant 1$, we have

$$
\operatorname{Bel}_{n, \lambda}(x+y)-\operatorname{Bel}_{n, \lambda}(x)=\sum_{k=1}^{n} \sum_{m=1}^{k} \sum_{l=1}^{m} \sum_{j=0}^{l-1}\left(\begin{array}{c}
m+1 \\
l
\end{array}\right)\left(\begin{array}{l}
l \\
j
\end{array}\right) S_{2}(k, m) S_{1}(n, k) \frac{\lambda^{n-k}}{m+1} y^{l-j} B_{j}(x) .
$$

Let

$$
\mathbb{P}_{n}=\{p(x) \in \mathbb{C}[x] \mid \operatorname{deg} p(x) \leqslant n\},(n \geqslant 0) .
$$

For $p(x) \in \mathbb{P}_{n}$, we assume that

$$
p(x)=\sum_{l=0}^{n} a_{l} \operatorname{Bel}_{l, \lambda}(x)
$$

From (2.2), we have

$$
\left\langle\left(\frac{(t+1)^{\lambda}-1}{\lambda}\right)^{m} \mid \operatorname{Bel}_{n, \lambda}(x)\right\rangle=n ! \delta_{m, n},(m, n \geqslant 0) .
$$


By (2.10) and (2.11), we get

$$
\left\langle\left(\frac{(t+1)^{\lambda}-1}{\lambda}\right)^{m} \mid p(x)\right\rangle=\sum_{l=0}^{n} a_{l}\left\langle\left(\frac{(t+1)^{\lambda}-1}{\lambda}\right)^{m} \mid \operatorname{Bel}_{l, \lambda}(x)\right\rangle=\sum_{l=0}^{n} a_{l} l ! \delta_{l, m}=m ! a_{m} .
$$

From (2.12), we have

$$
a_{m}=\frac{1}{m !}\left\langle\left(\frac{(t+1)^{\lambda}-1}{\lambda}\right)^{m} \mid p(x)\right\rangle,(m \geqslant 0) .
$$

Therefore, by (2.10) and (2.13), we obtain the following theorem.

Theorem 2.4. For $p(x) \in \mathbb{P}_{n},(n \geqslant 0)$, we have

$$
p(x)=\sum_{m=0}^{n} a_{m} \operatorname{Bel}_{m, \lambda}(x)
$$

where

$$
a_{m}=\frac{1}{m !}\left\langle\left(\frac{(t+1)^{\lambda}-1}{\lambda}\right)^{m} \mid p(x)\right\rangle
$$

Let us take $p(x)=B_{n}(x) \in \mathbb{P}_{n},(n \geqslant 0)$. Then, by Theorem 2.4, we get

$$
B_{n}(x)=\sum_{m=0}^{n} a_{m} \operatorname{Bel}_{m, \lambda}(x),
$$

where

$$
\begin{aligned}
a_{m} & =\frac{1}{m !}\left\langle\left(\frac{(1+t)^{\lambda}-1}{\lambda}\right)^{m} \mid B_{n}(x)\right\rangle=\frac{\lambda^{-m}}{m !} \sum_{l=0}^{m}\left(\begin{array}{c}
m \\
l
\end{array}\right)(-1)^{m-l}\left\langle(1+t)^{\lambda l} \mid B_{n}(x)\right\rangle \\
& =\frac{\lambda^{-m}}{m !} \sum_{l=0}^{m} \sum_{k=0}^{\infty}\left(\begin{array}{c}
m \\
l
\end{array}\right)\left(\begin{array}{c}
\lambda l \\
k
\end{array}\right)(-1)^{m-l}\left\langle t^{k} \mid B_{n}(x)\right\rangle \\
& =\frac{\lambda^{-m}}{m !} \sum_{l=0}^{m} \sum_{k=0}^{n}\left(\begin{array}{c}
m \\
l
\end{array}\right)\left(\begin{array}{c}
\lambda l \\
k
\end{array}\right)(-1)^{m-l}(n)_{k} B_{n-k} \\
& =\frac{\lambda^{-m}}{m !} \sum_{l=0}^{m} \sum_{k=0}^{n}\left(\begin{array}{c}
m \\
l
\end{array}\right)\left(\begin{array}{c}
\lambda l \\
k
\end{array}\right)\left(\begin{array}{c}
n \\
k
\end{array}\right)(-1)^{m-l} B_{n-k} k ! .
\end{aligned}
$$

We have another expression for $a_{m}$.

$$
\begin{aligned}
a_{m} & =\lambda^{-m}\left\langle\frac{1}{m !}\left((1+t)^{\lambda}-1\right)^{m} \mid B_{n}(x)\right\rangle \\
& =\lambda^{-m}\left\langle\frac{1}{m !}\left(e^{\lambda \log (1+t)}-1\right)^{m} \mid B_{n}(x)\right\rangle \\
& =\lambda^{-m} \sum_{k=m}^{n} S_{2}(k, m) \lambda^{k}\left\langle\frac{1}{k !}(\log (1+t))^{k} \mid B_{n}(x)\right\rangle \\
& =\sum_{k=m}^{n} S_{2}(k, m) \lambda^{k-m}\left\langle\sum_{j=k}^{n} S_{1}(j, k) \frac{t^{j}}{j !} \mid B_{n}(x)\right\rangle \\
& =\sum_{k=m}^{n} \sum_{j=k}^{n} S_{2}(k, m) S_{1}(j, k) \lambda^{k-m}\left(\begin{array}{l}
n \\
j
\end{array}\right) B_{n-j} .
\end{aligned}
$$

Therefore, by (2.14), (2.15), and (2.16), we obtain the following theorem. 
Theorem 2.5. For $\mathrm{n} \geqslant 0$, we have

$$
\begin{aligned}
B_{n}(x) & =\sum_{m=0}^{n}\left(\sum_{l=0}^{m} \sum_{k=0}^{n}\left(\begin{array}{c}
m \\
l
\end{array}\right)\left(\begin{array}{c}
\lambda l \\
k
\end{array}\right)\left(\begin{array}{l}
n \\
k
\end{array}\right) \lambda^{-m}(-1)^{m-l} \frac{k !}{m !} B_{n-k}\right) \operatorname{Bel}_{m, \lambda}(x) \\
& =\sum_{m=0}^{n}\left(\sum_{k=m}^{n} \sum_{j=k}^{n} S_{2}(k, m) S_{1}(j, k) \lambda^{k-m}\left(\begin{array}{c}
n \\
j
\end{array}\right) B_{n-j}\right) \operatorname{Bel}_{m, \lambda}(x) .
\end{aligned}
$$

Let us consider the following two Sheffer sequences:

$$
\operatorname{Bel}_{n, \lambda}(x) \sim\left(1, \frac{(1+t)^{\lambda}-1}{\lambda}\right), \quad x^{n} \sim(1, t), \quad(n \geqslant 0) .
$$

Then, by (1.13), (1.14), and (2.17), we get

$$
\operatorname{Bel}_{n, \lambda}(x)=\sum_{m=0}^{n} C_{n, m} x^{m}
$$

where

$$
\begin{aligned}
C_{n, m} & =\frac{1}{m !}\left\langle\left((\lambda t+1)^{\frac{1}{\lambda}}-1\right)^{m} \mid x^{n}\right\rangle \\
& =\frac{1}{m !} \sum_{l=0}^{m}\left(\begin{array}{c}
m \\
l
\end{array}\right)(-1)^{m-l}\left\langle(\lambda t+1)^{\frac{l}{\lambda}} \mid x^{n}\right\rangle \\
& =\frac{1}{m !} \sum_{l=0}^{m}\left(\begin{array}{c}
m \\
l
\end{array}\right)(-1)^{m-l} \sum_{k=0}^{\infty}\left(\begin{array}{c}
\frac{l}{\lambda} \\
k
\end{array}\right) \lambda^{k}\left\langle t^{k} \mid x^{n}\right\rangle \\
& =\frac{n !}{m !} \sum_{l=0}^{m}\left(\begin{array}{c}
m \\
l
\end{array}\right)\left(\begin{array}{c}
\frac{l}{\lambda} \\
n
\end{array}\right)(-1)^{m-l} \lambda^{n} .
\end{aligned}
$$

Therefore, by (2.18) and (2.19), we obtain the following theorem.

Theorem 2.6. For $\mathrm{n} \geqslant 0$, we have

$$
\operatorname{Bel}_{n, \lambda}(x)=n ! \lambda^{n} \sum_{m=0}^{n}\left(\sum_{l=0}^{m} \frac{1}{m !}\left(\begin{array}{c}
m \\
l
\end{array}\right)\left(\begin{array}{c}
\frac{l}{\lambda} \\
n
\end{array}\right)(-1)^{m-l}\right) x^{m} .
$$

Remark 2.7. Note that

$$
\lambda^{n}\left(\begin{array}{l}
\frac{l}{\lambda} \\
n
\end{array}\right)=\frac{l(l-\lambda)(l-2 \lambda) \cdots(l-(n-1) \lambda)}{n !},(n \geqslant 0)
$$

By (2.20), we easily get

$$
\lim _{\lambda \rightarrow 0} \lambda^{n}\left(\begin{array}{l}
\frac{l}{\lambda} \\
n
\end{array}\right)=\frac{l^{n}}{n !},(n \geqslant 0) .
$$

From Theorem 2.6, we have

$$
\begin{aligned}
\lim _{\lambda \rightarrow 0} \operatorname{Bel}_{n, \lambda}(x) & =\sum_{m=0}^{n}\left(\sum_{l=0}^{m} \frac{1}{m !}\left(\begin{array}{c}
m \\
l
\end{array}\right) l^{n}(-1)^{m-l}\right) x^{m} \\
& =\sum_{m=0}^{n}\left(\frac{1}{m !} \Delta^{m} 0^{n}\right) x^{m}=\sum_{m=0}^{n} S_{2}(n, m) x^{m}=\operatorname{Bel}_{n}(x),
\end{aligned}
$$


where $\Delta$ is the difference operator with $\Delta f(x)=f(x+1)-f(x)$.

For $(x)_{n} \sim\left(1, e^{t}-1\right)$, we have

$$
\begin{aligned}
e^{x \log (1+t)}=\sum_{m=0}^{\infty} x^{m} \frac{1}{m !}(\log (1+t))^{m} & =\sum_{m=0}^{\infty} x^{m} \sum_{n=m}^{\infty} S_{1}(n, m) \frac{t^{n}}{n !} \\
& =\sum_{n=0}^{\infty}\left(\sum_{m=0}^{n} S_{1}(n, m) x^{m}\right) \frac{t^{n}}{n !}=\sum_{n=0}^{\infty}(x)_{n} \frac{t^{n}}{n !} .
\end{aligned}
$$

Let us consider the following two Sheffer sequences:

$$
\operatorname{Bel}_{n, \lambda}(x) \sim\left(1, \frac{(1+t)^{\lambda}-1}{\lambda}\right), \quad(x)_{n} \sim\left(1, e^{t}-1\right) .
$$

From (1.13), (1.14), and (2.21), we have

$$
\operatorname{Bel}_{n, \lambda}(x)=\sum_{m=0}^{n} C_{n, m}(x)_{m},(n \geqslant 0) .
$$

where

$$
\begin{aligned}
C_{n, m} & =\frac{1}{m !}\left\langle\left(e^{\left((\lambda t+1)^{\frac{1}{\lambda}}-1\right)}-1\right)^{m} \mid x^{n}\right\rangle \\
& =\sum_{k=m}^{\infty} S_{2}(k, m) \frac{1}{k !}\left\langle\left((\lambda t+1)^{\frac{1}{\lambda}}-1\right)^{k} \mid x^{n}\right\rangle \\
& =\sum_{k=m}^{n} \frac{S_{2}(k, m)}{k !} \sum_{l=0}^{k}\left(\begin{array}{l}
k \\
l
\end{array}\right)(-1)^{k-l}\left\langle(\lambda t+1)^{\frac{l}{\lambda}} \mid x^{n}\right\rangle \\
& =\sum_{k=m}^{n} \frac{S_{2}(k, m)}{k !} \sum_{l=0}^{k}\left(\begin{array}{l}
k \\
l
\end{array}\right)(-1)^{k-l} \sum_{j=0}^{\infty}\left(\begin{array}{l}
\frac{l}{\lambda} \\
j
\end{array}\right) \lambda^{j}\left\langle t^{j} \mid x^{n}\right\rangle \\
& =\sum_{k=m}^{n} \frac{S_{2}(k, m)}{k !} \sum_{l=0}^{k}\left(\begin{array}{l}
k \\
l
\end{array}\right)(-1)^{k-l}\left(\begin{array}{l}
\frac{l}{\lambda} \\
n
\end{array}\right) \lambda^{n} n ! \\
& =n ! \lambda^{n} \sum_{k=m}^{n} \sum_{l=0}^{k} \frac{S_{2}(k, m)}{k !}\left(\begin{array}{l}
k \\
l
\end{array}\right)\left(\begin{array}{l}
\frac{l}{\lambda} \\
n
\end{array}\right)(-1)^{k-l} .
\end{aligned}
$$

Therefore, by (2.22) and (2.23), we obtain the following theorem.

Theorem 2.8. For $n \geqslant 0$, we have

$$
\operatorname{Bel}_{n, \lambda}(x)=n ! \lambda^{n} \sum_{m=0}^{n}\left(\sum_{k=m}^{n} \sum_{l=0}^{k} \frac{s_{2}(k, m)}{k !}\left(\begin{array}{l}
k \\
l
\end{array}\right)\left(\begin{array}{l}
\frac{l}{\lambda} \\
n
\end{array}\right)(-1)^{k-l}\right)(x)_{m} .
$$

On the other hand,

$$
\begin{aligned}
C_{n, m} & =\frac{1}{m !}\left\langle\left(e^{\left((\lambda t+1)^{\frac{1}{\lambda}}-1\right)}-1\right)^{m} \mid x^{n}\right\rangle, \\
\left(e^{\left((\lambda t+1)^{\frac{1}{\lambda}}-1\right)}-1\right)^{m} & =\underbrace{\left(e^{\left((\lambda t+1)^{\frac{1}{\lambda}}-1\right)}-1\right) \times \cdots \times\left(e^{\left((\lambda t+1)^{\frac{1}{\lambda}}-1\right)}-1\right)}_{m-\text { times }} \\
& =\left(\sum_{l_{1}=1}^{\infty} \operatorname{Bel}_{l_{1}, \lambda} \frac{t^{l_{1}}}{l_{1} !}\right) \times \cdots \times\left(\sum_{l_{m}=1}^{\infty} \operatorname{Bel}_{l_{m}, \lambda} \frac{t^{l_{m}}}{l_{m} !}\right)
\end{aligned}
$$




$$
=\sum_{k=m}^{\infty}\left(\sum_{\substack{l_{1}+\ldots+l_{m}=k \\
l_{1}, \cdots, l_{m} \geqslant 1}}\left(\begin{array}{c}
k \\
l_{1}, \cdots, l_{m}
\end{array}\right) \operatorname{Bel}_{l_{1}, \lambda} \cdots \operatorname{Bel}_{l_{m}, \lambda}\right) \frac{t^{k}}{k !} .
$$

From (2.24) and (2.25), we have

$$
\begin{aligned}
& C_{n, m}=\frac{1}{m !}\left\langle\left(e^{\left((\lambda t+1)^{\frac{1}{\lambda}}-1\right)}-1\right)^{m} \mid x^{n}\right\rangle \\
& =\frac{1}{m !} \sum_{k=m}^{n}\left(\sum_{\substack{l_{1}+\cdots+l_{m}=k \\
l_{1}, \cdots, l_{m} \geqslant 1}}\left(\begin{array}{c}
k \\
l_{1}, \cdots, l_{m}
\end{array}\right) \operatorname{Bel}_{l_{1}, \lambda} \cdots \operatorname{Bel}_{l_{m}, \lambda}\right) \frac{1}{k !}\left\langle t^{k} \mid x^{n}\right\rangle \\
& =\frac{1}{m !} \sum_{\substack{l_{1}+\cdots, l_{m}=n \\
l_{1}, \cdots, l_{m} \geqslant 1}}\left(\begin{array}{c}
n \\
l_{1}, \cdots, l_{m}
\end{array}\right) \operatorname{Bel}_{l_{1}, \lambda} \cdots \operatorname{Bel}_{l_{m}, \lambda} .
\end{aligned}
$$

Therefore, by (2.22) and (2.26), we get

$$
\operatorname{Bel}_{n, \lambda}(x)=\sum_{m=0}^{n}\left(\frac{1}{m !} \sum_{\substack{l_{1}+\cdots+l_{m}=n \\
l_{1}, \cdots, l_{m} \geqslant 1}}\left(\begin{array}{c}
n \\
l_{1}, \cdots, l_{m}
\end{array}\right) \operatorname{Bel}_{l_{1}, \lambda} \cdots \operatorname{Bel}_{l_{m}, \lambda}\right)(x)_{m} .
$$

\section{References}

[1] H. W. Becker, J. Riordan, The arithmetic of Bell and Stirling numbers, Amer. J. Math., 70 (1948), 385-394. 1

[2] F. Brafman, On Touchard polynomials, Canad. J. Math., 9 (1957), 191-193.

[3] L. Carlitz, Congruences for generalized Bell and Stirling numbers, Duke Math. J., 22 (1955), 193-205.

[4] L. Carlitz, Some polynomials of Touchard connected with the Bernoulli numbers, Canad. J. Math., 9 (1957), 188-190. 1

[5] L. Carlitz, Degenerate Stirling, Bernoulli and Eulerian numbers, Utilitas Math., 15 (1979), 51-88.

[6] L. Carlitz, Some remarks on the Bell numbers, Fibonacci Quart., 18 (1980), 66-73.

[7] Y. He, J. Pan, Some recursion formulae for the number of derangements and Bell numbers, J. Math. Res. Appl., 36 (2016), 15-22. 1

[8] L.-C. Jang, T. Kim, Some identities of Bell polynomials, J. Comput. Anal. Appl., 20 (2016), 584-589.

[9] J. B. Kim, On some numbers from the Bell polynomials, Math. Japon., 18 (1973), 1-4.

[10] D. S. Kim, T. Kim, Some identities of Bell polynomials, Sci. China Math., 58 (2015), 2095-2104. 1, 1

[11] D. S. Kim, T. Kim, Umbral calculus associated with Bernoulli polynomials, J. Number Theory, 147 (2015), 871-882. 1, $1,1,1$

[12] D. S. Kim, T. Kim, On degenerate Bell numbers and polynomials, Rev. R. Acad. Cienc. Exactas F11s. Nat. Ser. A Math. RACSAM, 111 (2016), 435-446. 1

[13] D. S. Kim, T. Kim, On partially degenerate Bell numbers and polynomials, (communicated). 1

[14] T. Kim, T. Mansour, Umbral calculus associated with Frobenius-type Eulerian polynomials, Russ. J. Math. Phys., 21 (2014), 484-493. 1, 1, 1, 1

[15] S.-H. Rim, H. K. Pak, J. K. Kwon, T. G. Kim, Some identities of Bell polynomials associated with p-adic integral on $\mathbb{Z}_{\mathrm{p}}$, J. Comput. Anal. Appl., 20 (2016), 437-446.

[16] S. Roman, The umbral calculus, Pure and Applied Mathematics, Academic Press, Inc. [Harcourt Brace Jovanovich, Publishers], New York, (1984). 1, 1, 1, 1, 1.6, 1.9, 1

[17] M. Wyman, L. Moser, On some polynomials of Touchard, Canad. J. Math., 8 (1956), 321-322. 1 